\title{
An observational study of implicit motor imagery using laterality recognition of the hand after stroke
}

\author{
Marsden, Jonathan
}

http://hdl.handle.net/10026.1/4987

\subsection{9/02699052.2016.1147600}

Brain Injury

Informa UK Limited

All content in PEARL is protected by copyright law. Author manuscripts are made available in accordance with publisher policies. Please cite only the published version using the details provided on the item record or document. In the absence of an open licence (e.g. Creative Commons), permissions for further reuse of content should be sought from the publisher or author. 
1 This is an accepted article published by Brain Injury available at

2 http://www.tandfonline.com/doi/abs/10.3109/02699052.2016.1147600?journalCode=i

3 bij20

$4 \quad$ DOI 10.3109/02699052.2016.1147600

5

6

7

8

9

10

11

12

13 Title: An observational study of implicit motor imagery using laterality recognition of 14 the hand after stroke

15

16

17

18

19

20

21

22 
5 Abstract

6 Objective: To explore the relationship between laterality recognition after stroke and 7 impairments in attention, 3D object rotation and functional ability.

8 Design: Observational cross-sectional study

9 Setting: Acute care teaching hospital

10 Participants: Thirty-two acute and sub-acute people with stroke and 36 healthy, 11 age-matched controls.

12 Interventions: Not applicable

13 Main Outcome Measures: Laterality recognition, attention and mental rotation of objects. Within the stroke group, the relationship between laterality recognition and functional ability, neglect, hemianopia and dyspraxia were further explored.

Results: People with stroke were significantly less accurate (69\% vs $80 \%)$ and showed delayed reaction times (3.0secs vs $1.9 \mathrm{secs}$ ) when determining the laterality of a pictured hand. Deficits either in accuracy or reaction times were seen in $53 \%$ of people with stroke. The accuracy of laterality recognition was associated with reduced functional ability $\left(R^{2}=0.21\right)$, less accurate mental rotation of objects

$21 \quad\left(\mathrm{R}^{2}=0.20\right)$ and dyspraxia $(p=0.03)$.

22 Conclusion: Implicit motor imagery is affected in a significant number of patients after stroke with these deficits related to lesions to the motor networks as well as other deficits seen after stroke. This research provides new insights into how laterality recognition is related to a number of other deficits after stroke, including the mental rotation of 3D objects, attention and dyspraxia. Further research is required to determine if treatment programs can improve deficits in laterality recognition and impact functional outcomes after stroke.

Key Words: stroke, perception, attention 


\section{Abbreviations:}

2 Connor's Continuous Performance Test II (CCPT)

3 Hand Laterality Recognition Task (HLRT),

4 Catherine Bergego Scale (CBS).

$5 \quad$ Modified Barthel Index (MBI)

6

7

8 
1 Stroke is a leading cause of disability worldwide [1] , with over one third of survivors continuing to be functionally dependent a year after the stroke [2]. Only $12 \%$ of people with stroke with an initial upper limb motor deficit achieve complete functional recovery, while $38 \%$ regain some dexterity in the affected upper limb [3]. Understanding factors affecting functional deficits will aid in the targeting of future interventions.

Motor imagery can be divided into explicit motor imagery or implicit motor imagery[4]. Explicit motor imagery, or mental practice has been shown to be an effective treatment for the upper limb when combined with conventional therapy after stroke [5] [6-8]. Implicit motor imagery is most commonly assessed by the Hand Laterality Recognition Task (HLRT), which involves identifying whether pictures of a body part are left or right [4, 9-11]

Deficits in laterality recognition have been shown after a stroke [4, 12-20]. After a stroke, participants are less accurate [4] and slower in the hand laterality recognition task [13] however, even with severe hemiparesis, participants are able to use implicit motor imagery to solve a task [19]. Motor imagery recruits the same neural networks as those involved in movement execution [21] including the supplementary motor area, prefrontal cortex, premotor cortex, parietal cortex, cingulate cortex, cerebellum and inferior frontal cortex [8,21]. Given the common neural networks involved in laterality recognition and motor planning/production, deficits in laterality recognition may be associated with deficits in functional movements although this relationship has not been explored to date.

Laterality recognition relies on the mental rotation of hands to solve the task and has been compared to the ability to mentally rotate objects using visual imagery after stroke. Using fMRI Kosslyn et al (1998) showed separate neural processes in these 2 tasks; the rotation of hands recruited motor processing areas, whereas the rotation of objects recruited visual processing areas [22]. A double dissociation between the ability to perform motor and visual imagery has been shown in some studies with people with stroke specifically selected according to the area of damage $[14,16,17]$. However, De Vries et al (2011) showed that people with stroke may have impairments in motor imagery, visual imagery or both at 3 or 6 weeks post stroke [4]. 
1 As reported in other patient groups (eg amputees [23]) it may be that people with stroke use a visual transformation of the stimuli rather that motor imagery to solve the laterality recognition task. Impaired laterality recognition could also be associated with other deficits that are present after stroke, such as neglect or attention. Vromen et al (2011) found that in 20 participants with right hemispheric lesions, patients with neglect did not perform as well in a hand laterality recognition task as patients without neglect [12]. They also found that the group with neglect scored significantly less on the tests of attention, however it was not mentioned if the attention impairment correlated with the impairment in motor imagery ability.

In summary, the relationship between a stroke and laterality recognition remains unclear. There is limited evidence about how laterality recognition after a stroke is related to visual imagery and the presence of other deficits such as neglect or sustained attention. Finally, the relationship between laterality deficits and function has not been explored to date.

Laterality deficits are seen in other conditions such as chronic regional pain syndromes where the deficits are felt to reflect brain re-organisation over time. In such conditions training of laterality recognition in combination with other techniques such as motor imagery lead to reductions in the primary impairment (pain) and improvements in hand function[24]. If laterality recognition is related to impaired function following a stroke it could be that similar interventions could be trialled in the future with the aim of improving motor control and functional ability.

The aims of the study are to (a) investigate the relationship between laterality recognition and potential causative factors such as impaired ability to mentally rotate objects or to sustain attention on a task; and (b) investigate the relationship between laterality deficits and functional ability.

\section{Methods}

A prospective observational cross-section design compared laterality recognition ability in people with a stroke and a healthy age-matched control group. 
1 Inclusion criteria included radiological evidence of a stroke within the last 12 months and the ability to provide informed consent to participate in the study. Major exclusion criteria included previous stroke, pre-existing cognitive impairments, or patients who were medically unstable. Prospective sampling occurred with consecutive people with a stroke admitted to the stoke or the rehabilitation unit at a metropolitan hospital assessed.

The study was approved by the relevant Health Service Human Research Ethics Committee as well as Plymouth University, Faculty of Health, Education and Society Health Student Ethics Sub-Committee. All participants were provided with an information sheet and signed a consent form to participate in the study.

\section{Hand Laterality Recognition}

Implicit motor imagery ability was assessed using a hand laterality task (Recognise program, Neuro Orthopaedic Institute, 2011). Colour photographs of a hand in different positions were displayed on the screen and the subject responded by pressing the 'a' key for a left hand image or the 'd' key for a right hand image on a keyboard. The images were displayed on the screen until the subject responded up to a maximum of 10 seconds. Participants completed 4 blocks of 30 images randomly selected from the bank of 197 images in the program. The accuracy (percentage of correct responses) and reaction times were analysed. Reaction times below $150 \mathrm{~ms}$ were excluded from the data (3\% of responses).

\section{Mental Rotation of 3D objects}

Two pictures of Shepard-Metzler objects [25] that were rotations and/or reflections of a 10-block base object were displayed using Psychopy2. The participants responded with the 'a' key if the two objects were the same and the ' $d$ ' key if the objects were different. Each pair of 3D objects was shown for a maximum of 7 seconds. Eighty unique pairs of images were shown from a bank of 15 rotations of the base object.

\section{Sustained Attention}

Sustained attention was assessed using the computerised Connor's Continuous Performance Test II (CCPT) (Version 5, Multi-Health Systems Inc). Participants responded by pressing the space bar on a keyboard when letters, except for the 
1 letter ' $\mathrm{X}$ ', were displayed on the screen. A practice session was completed for 70

2 seconds prior to the 14 minute assessment period. Reaction time and a composite attention score ("Confidence Index") was calculated to determine the percentage likelihood that the subject fits a clinical picture of attention deficits or a non-clinical

5 picture.

\section{Clinical assessment of deficits}

7 Neglect

8 The presence of neglect was assessed using the Catherine Bergego Scale

9 (CBS). This scale has been found to be valid and reliable in sub-acute and chronic people with a stroke, and correlates to, but is more sensitive than conventional pen and paper tests of neglect [26]

\section{Function}

Function was measured using the Modified Barthel Index (MBI) which is an adapted version of the Barthel Index with improved sensitivity and reliability [27].

\section{Lesion location and other deficits}

The side of the lesion and lesion location was determined from MRI scans and reports. Documentation of hemianopia, dysphasia and dyspraxia was sourced from the medical record.

\section{Procedure}

The computer based assessments commenced with the CCPT, the laterality recognition assessment, and then the $3 \mathrm{D}$ object rotation. During the assessments participants sat supported in a chair with the arm that was not involved in the assessment positioned supported with the palm down. To reduce the impact of dysphasia, written cue cards were provided underneath the monitor with the letter and response key for the assessments for laterality recognition and 3D rotation. People with a stroke responded with their unaffected hand, which was defined as the hand ipsilateral to a cerebral/hemisphere stroke or contralateral to a medullary or cerebellar stroke, even if they did not have any residual weakness/incoordination. Images were presented in the spared visual hemifield for people with hemianopia.Control participants responded with the hand indicated by the researcher; a similar proportion of dominant/non-dominant response hands to the 
1 stroke group were selected. Clinical assessments were completed within 2 days

2 following the computer based assessment.

4 Sample Size

5 Sample size was based on Reinersmann et al. (2009) who assessed laterality recognition in Chronic Regional pain syndrome. Reactions times in the chronic pain group $(2.3 s \pm 0.8)$ were significantly longer than the healthy controls (1.8s $\pm 0.5 \mathrm{~s})$. Assuming a similar effect size (0.73) when comparing the stroke group with controls, a sample size of 30 in each group (stroke and healthy controls) was required (power $=0.8, \alpha=0.05$ )

\section{Data Analysis}

Statistics were analysed using SPSS 18.0. Group differences were examined unpaired t-tests (e.g. age, 3D rotation, attention reaction time) or chi-squared tests(e.g. gender and hand dominance )

Three separate between group (stroke vs. control) repeated measures analysis of variances (ANOVA) for laterality recognition accuracy and reaction time were performed. The laterality of the pictured hand were separately analysed using the factors left vs. right hand; dominant vs. non-dominant hand; affected vs. unaffected hand. To analyse the data for pictures of the affected vs. unaffected, the unaffected hand was considered to be the response hand, with control participants analysed in the same way.

Participants with deficits in laterality recognition accuracy or reaction time, 3D object rotation and reaction time for attention were defined by scores that were 2 standard deviations below the mean for the control group. Participants were defined as having a deficit if they scored greater than 0 on the CBS for neglect.

Correlations were calculated between laterality recognition accuracy and reaction times with attention reaction time and 3D rotation accuracy with Pearson's correlation co-efficient. As the data was not normally distributed, a Spearman's correlation coefficient explored the relationship with function. Data is summarised as the mean \pm standard deviation; significance is taken as $p \leq 0.05$ 
2 Results

\section{Participants}

4 Over a 6 month period 121 patients were admitted of these 34 patients fit the

5 inclusion criteria. One patient was discharged prior to being invited to participate in

6 the research, 33 consented to the research, but 1 patient withdrew after completing

7 the practice for the attention assessment. Thirty-two people with a stroke (mean age

$8=64.9 \pm 13$ ) and 36 control participants (mean age $=61.1 \pm 15$ ) were therefore

9 assessed. Group characteristics are summarised in Table 1.

There was no significant difference between the stroke and control group for age $(t(66)=1.10 p=0.28)$. There was a significant difference between the stroke and control group for gender $(p=0.006)$ with more males in the control group and more females in the stroke group. There was no significant difference for dominant hand $(p=0.78)$, with the majority of participants reporting right hand dominance. In the stroke group the average time since stroke was $18.2 \pm 29$ days with a range of 2 to 135 days and a median of 7 days. $81 \%$ of people with a stroke were assessed less than 3 weeks post stroke. The characteristics of people with a stroke are shown in Table 2.

\section{Group Analysis}

\section{Laterality recognition accuracy}

There was a significant effect of group $(F(1,66)=14.7, p<0.001)$ with the mean accuracy scores lower for the stroke group $(68.6 \% \pm 15 \%)$ than the control group $(80.1 \% \pm 12 \%$, table 3$)$. There was no significant difference between recognising the laterality of a picture of the left or right hand, dominant or non-dominant hand, or affected or unaffected hand and there were no significant interaction effects. Ten (31.3\%) participants in the stroke group were below the cut off value for laterality recognition accuracy of $57.4 \%$.

\section{Laterality recognition reaction times}

Reaction times for laterality recognition were slower in the stroke group (2.96 seconds $\pm 1.4 \mathrm{secs})$ than the control group (1.86secs $\pm 1.05 \mathrm{secs}, F(1,66)=14.1$, $p=0.001$, table 3 ). There was a significant effect of hand when recognising pictures 
1 of left vs. right hand $(F(1,66)=8.10, p=0.006)$ and dominant vs. non-dominant hand

$2(\mathrm{~F}(1,66)=6.0, \mathrm{p}=0.02)$. Faster reactions times were seen with the right and dominant

3 hand. There was no difference between affected vs. unaffected hand $(F(1,66)=0.56$,

$4 \quad \mathrm{p}=0.81)$. There were no significant interactions for hand*group for each of the above

5 analyses (left vs. right: $(F(1,66)=0.01, p=0.93)$; dominant vs. non-dominant:

$6(F(1,66)=0.24, p=0.62)$ and affected vs. unaffected: $(F(1,66)=2.61, p=0.11)$. Eleven

7 participants (34\%) were below the cut-off value for deficits in laterality recognition

8 reaction time $(3.95 \mathrm{secs})$.

\section{3D object rotation}

10 There was a significant difference in the accuracy of mental rotation of 3D objects $11(t(62)=2.50, p=0.015)$, with the stroke participants significantly less accurate than the control participants (stroke: $46 \% \pm 16 \%$; control: $56 \% \pm 14 \%$ ). Three participants $(9.4 \%)$ in the stroke group were below the $26.9 \%$ cut off score for deficits in the mental rotation of $3 \mathrm{D}$ objects.

\section{Attention}

Fifteen (47\%) stroke participants but none of the 36 control participants had a $99.9 \%$ confidence index for fitting a clinical profile for attention deficits on the CCPT. There was a significant difference in reaction times in the attention test, $(t(50)=3.43$, $\mathrm{p}=0.001)$, withpeople with a stroke having significantly longer reaction times $(531 \mathrm{~ms}$ $\pm 121 \mathrm{~ms}$ ) than control participants $(444 \mathrm{~ms} \pm 78 \mathrm{~ms})$. Ten participants $(32.2 \%)$ in the stroke group had reaction times above the cut-off value.

\section{Clinically assessed deficits}

\section{Neglect}

Three of the people with a stroke scored above 0 on the CBS for neglect with scores of between 5 and 21 . The median score for neglect was 0 .

\section{Function}

Scores on the MBI ranged from 27 to 100 , with a median of 98 . Fifteen people with a stroke scored 100 out of 100 , indicating functional independence.

\section{Other clinical deficits}

Six participants had documented hemianopia from the medical records. Five participants had documented dysphasia. Expressive and receptive dysphasia was 
1 not recorded separately. Four participants had documented dyspraxia; all participants with dyspraxia also had dysphasia.

3 Relationship with lesion location

Lesion locations for individual participants with deficits in laterality recognition, and/or 3D object rotation were examined. The majority of those with deficits in laterality recognition had lesions involving the parietal lobe, frontal lobe or other parts of the motor network. Participants with laterality recognition deficits had both left $(n=7)$ and right $(n=10)$ brain damage. Participants with deficits in 3D object rotation had lesions in fronto-parietal areas or occipital cortex on the left $(n=1)$ and right $(n=2)$ sides .

\section{Correlations}

In the stroke group there was a significant correlation between accuracy and reaction times in the laterality recognition task $R^{2}=0.13, p=0.04$, with participants that had higher accuracy also having quicker reaction times. Participants whose laterality recognition was more accurate were more accurate on 3D object rotation (table 4 ). In the control group this correlation was not significant $R^{2}=0.10, p=0.06$. People with a stroke who were less accurate and slower in laterality recognition were more dependent in function (table 4).

\section{Relationship with other deficits}

The participants with dyspraxia were significantly less accurate in laterality recognition $(p=0.03)$. There were no significant differences in people with and without neglect, hemianopia or dysphasia. Only 1 of the 6 people with a hemianopia showed a deficit in object rotation, 1 showed impaired laterality recognition accuracy and 2 showed an impaired laterality recognition reaction time.

\section{Discussion}

In keeping with previous work laterality recognition deficits were present $\sim 33 \%$ of people post stroke $[4,12-14,19]$. Reaction times for laterality recognition showed a faster response time when recognising pictures of the dominant hand in both the stroke group and the control group [23, 28, 29] possibly reflecting differences in the efficiency of planning/ imagining dominant as opposed to non-dominant hand movements. 
1 There was no significant difference in people with a stroke in terms of their accuracy or reaction times for recognising pictures of their affected vs. unaffected hand, although response time to the affected hand were longer. Daprati et al (2010) showed that patients with left brain damage with severe motor impairment were significantly slower recognising their affected hand but were also slower than controls when recognising their unaffected hand. This is felt to reflect difficulties in planning and producing movements of the affected hand. The lack of difference between recognising pictures of the affected vs. unaffected hand in the present study could be due to the broad inclusion criteria as 15 people with a stroke scored 100 on the $\mathrm{MBI}$ and therefore were completely independent. Without a specific measure for motor impairment of the upper limb, such as the Fugl-Meyer test, correlations between the severity of motor impairment and differences between affected and unaffected limb cannot be determined.

The current study did not find a difference between laterality recognition between participants with LBD or RBD. Seven of the 17 participants with deficits in laterality recognition had LBD and 10 had RBD. Daprati et al (2011) found that participants with RBD were less accurate in laterality recognition tasks; however Schwoebel and Coslett (2005) also found that deficits in laterality recognition were not specific to LBD or RBD. Schwoebel and Coslett (2005) used similar inclusion criteria to the current study, ie not based on behavioural criteria or lesion location.

The primary aim of this study was to explore factors that could possibly explain any differences in laterality recognition seen in the stroke group. People with a stroke were significantly less accurate in the ability to mentally rotate 3D objects. These results need to be interpreted with caution as accuracy levels were close to chance (i.e. $50 \%$ ) for both groups suggesting the task had a floor effect. This may reflect task difficulty and an age-related decline in their mental rotation ability [30]. Age was significantly correlated with object rotation accuracy in both the control $\left(R^{2}=0.23\right.$, $p=0.004)$ and the stroke group $\left(R^{2}=0.14, p=0.048\right)$. Gender differences have also been shown on tasks involving the mental rotation of 3D objects, with males more accurate than females [31]. There was a significant gender difference between the control group and the stroke group with the control group having more females than males, which could have impacted on the slower rotation seen in the stroke 
1 group. Females in the control group had an average of $50 \%$ accuracy on mental rotation of 3D objects, and males had an average of $65 \%$.

3 The accuracy of laterality recognition was significantly correlated with accuracy of 3D object rotation as previously reported [4]. However, Tomasino et al (2003ab) showed a double dissociation with laterality recognition accuracy and 3D object rotation, suggesting that there are separate neural correlates for visual and motor imagery[16, 18]. One explanation is that participants used different strategies to solve the laterality recognition task. Curtze et al (2010) suggested that upper limb amputees may use a visual transformation of the stimuli rather that motor imagery, especially when the motor imagery strategy was more difficult [23] The visual strategy to solve the hand laterality task is slower [32] and therefore if participants were using a visual strategy they would have longer reaction times and be more likely to be identified as having a deficit in laterality recognition. This may explain the weak association between the two impairments in the current study.

There was a correlation between laterality recognition and functional ability. However, this study does not show that the relationship is causative. Future work should assess whether laterality recognition can be trained in people with a stroke and whether this results in an improvement in hand function. To date paradigms are available for training people to recognise hand laterality in other patient groups (eg Chronic regional pain syndrome[24]) and these may adopted as part of a future clinical trial.

\section{Study Limitations}

The tests were performed in a set order. This may mean that the later tests (eg 3D object rotation) may be subject to cognitive fatigue. Cognitive fatigue may be greater in people with stroke and therefore accentuate differences between the stroke and control groups. People with stroke were recruited over a long time frame (2-135 days). This covers a period where marked improvements in impairments and functional ability can occur due to factors such as resolution of oedema and adaptive changes. Future work could assess longitudinal changes in laterality recognition or assess groups of people at more discrete time periods post stroke to ascertain the natural recovery of this impairment. Other impairments seemed to be associated with a higher incidence of hand laterality deficits. Clinically defined dyspraxia was 
1 associated with a deficit in laterality recognition whilst 2 out of 3 participants

2 behavioural neglect had a laterality deficit. Both of these impairments affect systems

3 involved in sensori-motor planning. More in depth, objective, screening of these

4 impairments is required to further explore these relationships in the future.

5 Conclusion

6 Implicit motor imagery, as assessed by the accuracy and speed of laterality

7 recognition task was affected in $\sim 33 \%$ of patients after acute stroke. Laterality

8 recognition deficits were related to impaired mental rotation of 3D objects and

9 dyspraxia. There was a correlation between deficits in laterality recognition and

10 function.

Acknowledgements

14 We would like to thank the Neuro Orthopaedic Institute for the use of the recognize program for this study 
References

1. Donnan GA, Fisher M, Macleod M, Davis SM. Stroke. Lancet 2008;371(9624):1612-23.

2. Aziz NA, Leonardi-Bee J, Phillips M, Gladman JR, Legg L, Walker MF. Therapy-based rehabilitation services for patients living at home more than one year after stroke. Cochrane Database Syst Rev 2008(2):CD005952.

3. Kwakkel G, Kollen BJ, van der Grond J, Prevo AJ. Probability of regaining dexterity in the flaccid upper limb: impact of severity of paresis and time since onset in acute stroke. Stroke 2003;34(9):2181-2186.

4. de Vries S, Tepper M, Otten B, Mulder T. Recovery of motor imagery ability in stroke patients. Rehabil Res Pract 2011;2011:283840.

5. Barclay-Goddard RE, Stevenson TJ, Poluha W, Thalman L. Mental practice for treating upper extremity deficits in individuals with hemiparesis after stroke. Cochrane Database Syst Rev 2011(5):CD005950.

6. Langhorne P, Coupar F, Pollock A. Motor recovery after stroke: a systematic review. Lancet Neurol 2009;8(8):741-54.

7. Zimmermann-Schlatter A, Schuster C, Puhan MA, Siekierka E, Steurer J. Efficacy of motor imagery in post-stroke rehabilitation: a systematic review. J Neuroeng Rehabil 2008;5:8.

8. Sharma N, Pomeroy VM, Baron JC. Motor imagery: a backdoor to the motor system after stroke? Stroke 2006;37(7):1941-52. 
9. Moseley GL, Sim DF, Henry ML, Souvlis T. Experimental hand pain delays recognition of the contralateral hand--evidence that acute and chronic pain have opposite effects on information processing? Brain Res Cogn Brain Res $2005 ; 25(1): 188-94$.

10. Parsons LM. Imagined spatial transformation of one's body. J Exp Psychol Gen 1987;116(2):172-91.

11. Parsons LM. Temporal and kinematic properties of motor behavior reflected in mentally simulated action. J Exp Psychol Hum Percept Perform 1994;20(4):709-30.

12. Vromen A, Verbunt JA, Rasquin S, Wade DT. Motor imagery in patients with a right hemisphere stroke and unilateral neglect. Brain Inj 2011;25(4):387-93.

13. Daprati E, Nico D, Duval S, Lacquaniti F. Different motor imagery modes following brain damage. Cortex 2010;46(8):1016-30.

14. Conson M, Sacco S, Sara M, Pistoia F, Grossi D, Trojano L. Selective motor imagery defect in patients with locked-in syndrome. Neuropsychologia 2008;46(11):2622-8.

15. Schwoebel J, Friedman R, Duda N, Coslett HB. Pain and the body schema: evidence for peripheral effects on mental representations of movement. Brain 2001;124(Pt 10):2098-104.

16. Tomasino B, Toraldo A, Rumiati RI. Dissociation between the mental rotation of visual images and motor images in unilateral brain-damaged patients. Brain Cogn 2003;51(3):368-71. 
17. Tomasino B, Rumiati RI. Effects of strategies on mental rotation and hemispheric lateralization: neuropsychological evidence. J Cogn Neurosci $2004 ; 16(5): 878-88$

18. Tomasino B, Rumiati RI, Umilta CA. Selective deficit of motor imagery as tapped by a left-right decision of visually presented hands. Brain Cogn 2003;53(2):376-80.

19. Johnson $\mathrm{SH}$. Imagining the impossible: intact motor representations in hemiplegics. Neuroreport 2000;11(4):729-32.

20. Coslett HB. Evidence for a disturbance of the body schema in neglect. Brain Cogn 1998;37(3):527-44.

21. de Vries S, Mulder T. Motor imagery and stroke rehabilitation: a critical discussion. J Rehabil Med 2007;39(1):5-13.

22. Kosslyn SM, DiGirolamo GJ, Thompson WL, Alpert NM. Mental rotation of objects versus hands: neural mechanisms revealed by positron emission tomography. Psychophysiology 1998;35(2):151-61.

23. Curtze C, Otten B, Postema K. Effects of lower limb amputation on the mental rotation of feet. Exp Brain Res 2010;201(3):527-34.

24. Moseley GL. Graded motor imagery is effective for long-standing complex regional pain syndrome: a randomised controlled trial. Pain 2004;108(1-2):192-8.

25. Shepard RN, Metzler J. Mental rotation of three-dimensional objects. Science 1971;171(3972):701-3. 
26. Azouvi P, Olivier S, de Montety G, Samuel C, Louis-Dreyfus A, Tesio L. Behavioral assessment of unilateral neglect: study of the psychometric properties of the Catherine Bergego Scale. Arch Phys Med Rehabil 2003;84(1):51-7.

27. Shah S, Vanclay F, Cooper B. Improving the sensitivity of the Barthel Index for stroke rehabilitation. J Clin Epidemiol 1989;42(8):703-9.

28. Reinersmann A, Haarmeyer GS, Blankenburg M, Frettloh J, Krumova EK, Ocklenburg S, Maier C. Left is where the $L$ is right. Significantly delayed reaction time in limb laterality recognition in both CRPS and phantom limb pain patients. Neurosci Lett 2010;486(3):240-5.

29. Nico D, Daprati E, Rigal F, Parsons L, Sirigu A. Left and right hand recognition in upper limb amputees. Brain 2004;127(Pt 1):120-32.

30. Devlin AL, Wilson PH. Adult age differences in the ability to mentally transform object and body stimuli. Neuropsychol Dev Cogn B Aging Neuropsychol Cogn 2010;17(6):709-29.

31. Neubauer AC, Bergner S, Schatz M. Two- vs. three-dimensional presentation of mental rotation tasks: Sex differences and effects of training on performance and brain activation. Intelligence 2010;38(5):529-539.

32. Corradi-Dell'Acqua C, Tomasino B, Fink GR. What is the position of an arm relative to the body? Neural correlates of body schema and body structural description. J Neurosci 2009;29(13):4162-71. 